\title{
ANALYSIS OF OPTIMAL UNIFORM TWO-DIMENSIONAL QUANTIZATION FOR THE LAPLACE SOURCE OVER BENNETT'S INTEGRAL
}

\author{
Zoran H.Peric ${ }^{1}$, Marko Novkovic ${ }^{1}$, Dragan A. Stojanovic ${ }^{2}$ \\ ${ }^{1}$ The Faculty of Electronic Engineering, \\ Beogradska 14, 18000 Nis, SCG, +381 18529101 \\ ${ }^{2}$ Telekom Srbija A.D, Vozdova 11, 18000 Nis, SCG, +381 18292800
}

\begin{abstract}
In this paper, the analysis of Bennett's integral is given for uniform quantization for two-dimensional Laplace sources using granular distortion $\mathrm{Dg}$. The goal of this paper is to find a simple equation for distortion by solving Bennett's integral for uniform two-dimensional quantization. The analysis for optimal uniform quantization for two-dimensional Laplace sources is done.
\end{abstract}

Keywords: Bennett's integral, uniform polar quantization, normalized moment of inertia.

\section{Introduction}

Quantizers play an important role in the theory and practice of modern-day signal processing. The asymptotic optimal quantization problem, even for the simplest case - uniform scalar quantization, is actual nowadays [1]. The importance of using the rectangular cells and the optimal density (number) of points for product quantization and Gaussian source is considered in [2-3].

The simplest vector quantization is two-dimensional vector quantization $(\mathrm{k}=2)$. We will consider uniform quantization for two-dimensional Laplace sources with respect to distortion $(D)$, represents i.e. the meansquare error (MSE).

The first approximation to the long-time-averaged probability density function (pdf) of amplitudes is provided by Laplacian model. The probability density function of the difference signal for an image waveform follows the Laplacian function [4]. Laplace source is a model for speech [4].

In $[5,6]$ the analyses for asymptotic uniform twodimensional quantization and asymptotic uniform polar quantization are given. The analysis for optimal uniform polar quantization is given in [7]. The similar analysis for Laplace source is given here. Optimizations are done with respect to granular distortion $\mathrm{D}_{\mathrm{g}}$, i.e. the mean-square error (MSE). The goal of this paper is to find a simple equation for distortion by solving Bennett's integral for uniform quantization for two-dimensional Laplace sources. The analysis for uniform quantization for two-dimensional Laplace sources is done.

\section{Uniform Two-Dimensional Quantization for the Laplace Source}

Consider two independent identically distributed Laplace random variables $\left(x_{1}, x_{2}\right)$ with the zero mean and the unity variance. Probability density function (pdf) of a zero mean, unit variance Laplacian random variable is given as follows:

$$
f(x)=\frac{1}{\sqrt{2}} e^{-\sqrt{2}|x|}
$$

From this equation, the joint pdf function of two independent, identically distributed Laplace random variables $\left(x_{1}, x_{2}\right)$ can be written as:

$$
f_{1,2}\left(x_{1}, x_{2}\right)=\frac{1}{2} e^{-\sqrt{2}\left(\left|x_{1}\right|+\left|x_{2}\right|\right)}
$$

To simplify the vector quantizer, the Helmert transformation is applied to the source vector giving contours with constant probability densities. This orthogonal transformation is defined as:

$$
r=\frac{1}{\sqrt{2}}\left(\left|x_{1}\right|+\left|x_{2}\right|\right), u=\frac{1}{\sqrt{2}}\left(\left|x_{1}\right|-\left|x_{2}\right|\right)
$$

The obtained probability density function is:

$$
f(r, u)=\frac{1}{2} e^{-2 r}
$$

The uniform quantizer (amplitude range $\left[0, r_{\max }\right]$ ) is partitioned into $L$ concentric domains as shown in Fig. 1 . The number of cells in the $i$-th concentric domain is denoted by $N_{u}\left(m_{i}\right)$. Every concentric domain can be further partitioned into four subdomains of the same 
size and form, i.e. $N_{u}\left(m_{i}\right)=4 L_{i}$. The number $L_{i}$ is same for all the subdomains in the $i$-th domain. Every subdomain is divided into $m_{i}$ rectangular cells. An output point is placed in the centre of each cell. Coordinates of the $j$-th output point in the $i$-th domain in $r$ - $u$ coordinate system are $\left(m_{i}, \hat{u}_{i, j}\right)$.

The dimensions of $L_{i}$ cells are

$$
\begin{aligned}
& \Delta_{r}=r_{i+1}-r_{i}=\frac{r_{\max }}{L}, \\
& \Delta_{u}(i)=u_{i, j+1}-u_{i, j}=\frac{2 m_{i}}{L_{i}} .
\end{aligned}
$$

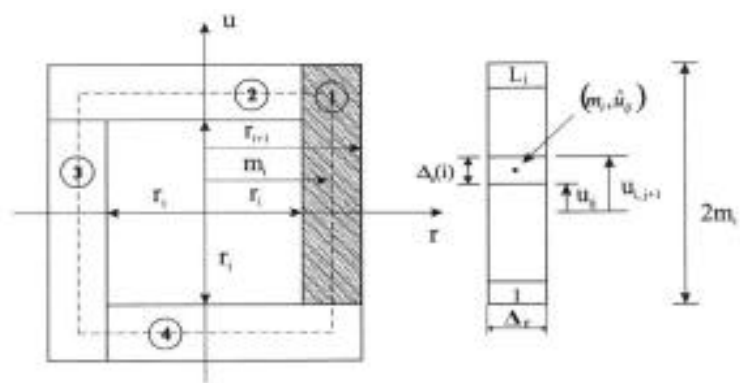

Figure 1. Two-dimensional space partitioning; the i-th concentric domain and the $\mathrm{j}$-th cell in the first subdomain

The coordinates of points are:

$$
\hat{u}_{i, j}=-r_{i}+(j-1 / 2) \Delta_{u}(i), \quad 1 \leq j \leq L_{i}
$$

where is: $r_{i}=i \Delta_{r}, \quad 1 \leq i \leq L, \quad r_{0}=0$

$$
m_{i}=(i-1 / 2) \Delta_{r}, 1 \leq i \leq L .
$$

Now, we will define granular distortion as

$$
D=D_{g}+D_{\text {over }},
$$

where $D_{g}$ is granular distortion and it is given by

$$
D_{g}=\sum_{i=1}^{L} \int_{r_{i}}^{r_{i+1}}\left[\left(r-m_{i}\right)^{2}+\frac{\left(\Delta_{u}(i)\right)^{2}}{12}\right] 2 m_{i} e^{-2 r} d r
$$

and $D_{\text {over }}$ is overload distorsion and it is given by

$$
D_{\text {over }}=\int_{r_{\max }}^{\infty}\left[\left(r-m_{L}\right)^{2}+\frac{\left(\Delta_{u}(L)\right)^{2}}{12}\right] 2 m_{L} e^{-2 r} d r
$$

\section{Bennett's Integral}

A two-dimensional N-point scalar quantizer is characterized by a partition $S=\left\{S_{1}, S_{2}, \ldots, S_{N}\right\}$ of twodimensional Euclidean space $R^{2}$ into $\mathrm{N}$ quantization cells and a code book, denoted as $C=\left\{\mathbf{y}_{1}, \mathbf{y}_{2}, \ldots, \mathbf{y}_{N}\right\}$, consisting of $N$ quantization points in two-dimensional Euclidean space $R^{2}$. A two-dimensional source vector $\mathbf{x}=\left\{x_{1}, x_{2}\right\}$ is quantized into one of the $\mathbf{y}_{i}$ 's according to the quantization rule $Q(\mathbf{x})=\mathbf{y}_{i}$ if $\mathbf{x} \in S_{i}$. The encoding rate for two-dimensional quantizer is $\log _{2}(N / 2)$. When applied to a random vector $\mathbf{x}=\left\{x_{1}, x_{2}\right\}$ with probability density function $p(\mathbf{x})$, quantizer's distortion is given by:

$$
D(S, C)=\frac{1}{2} \int\|\mathbf{x}-Q(\mathbf{x})\|^{2} p(\mathbf{x}) d \mathbf{x},
$$

that is,

$$
D(S, C)=\frac{1}{2} \sum_{i=1}^{N} \int_{S_{i}}\left\|\mathbf{x}-\mathbf{y}_{i}\right\|^{2} p(\mathbf{x}) d \mathbf{x},
$$

where $\left\|\mathbf{x}-\mathbf{y}_{i}\right\|$ denotes Euclidian distance:

$$
\left\|\mathbf{x}-\mathbf{y}_{\mathrm{i}}\right\|=\left(\sum_{j=1}^{2}\left(x_{j}-y_{i j}\right)^{2}\right)^{\frac{1}{2}}
$$

and $p(\mathbf{x})$ is the two-dimensional density of function $\mathbf{x}$.

The pioneering work of Bennett (9) showed that the mean-squared error of a scalar quantizer $(k=1)$, with many small cells ( $N$ large) and with each $y_{i}$ in the center of its cell may be accurately approximated as:

$$
D(S, C) \cong \frac{1}{12 N^{2}} \int \frac{1}{\lambda(x)^{2}} p(x) d x,
$$

where $\lambda(x)$ is a function, called point density, and $\lambda(x) \Delta$ is the fraction of quantization points in a small interval of width $\Delta$ surrounding $x$. The integral without limits denotes an integral over the entire space. The right-hand side of (9) is known as Bennett's integral. Althought originally derived for companders (quantizers consisting of a compressor mapping, uniform quantizer, and expander mapping) with $\lambda$ equal to the derivative of the compressor function can be recognized by other quantizers, and is applied more generally. Bennett's integral shows how the distortion depends on the key characteristics of the quantizer, namely, the number of points $N$ and a point density $\lambda$. Its utility is exemplified by the fact that one may use it to show that the best quantizers have:

$$
\lambda(x) \cong \frac{p(x)^{1 / 3}}{\int p(x)^{1 / 3} d x} .
$$

In the following analysis, we extend Bennett's integral to uniform quantization for two-dimensional Laplace sources. The goal is to give a simple approximate formula for distortion that would show influence of key characteristics.

Approximation of two-dimensional vector quantizer distortion over Bennett's integral is

$$
D_{g}=\frac{1}{N} \int \frac{m(r)}{\lambda(r)} p(r) d r
$$

where $m(r)$ is inertial profile.

The normalized moment of inertia is defined as:

$$
M\left(S, m_{i}\right)=\frac{1}{2} \frac{1}{\Delta_{r} \Delta_{u}(i)}\left(\frac{\Delta_{r}^{2}}{12}+\frac{\Delta_{u}^{2}(i)}{12}\right),
$$


where

$$
\Delta_{r}=\frac{r_{\max }}{L}, \Delta_{u}(i)=\frac{2 m_{i}}{L_{i}} .
$$

Using approximations $m_{i} \approx r$ and $m(r) \approx M\left(S, m_{i}\right)$, we find the moment of inertia as

$$
m(r)=\frac{1}{4} \frac{L L_{i}(r)}{r r_{\max }} \frac{1}{12}\left(\left(\frac{r_{\max }}{L}\right)^{2}+\left(\frac{2 r}{L_{i}(r)}\right)^{2}\right) .
$$

The point density function is given by

$$
\lambda(x) \equiv\left(N \operatorname{vol}\left(S_{x}\right)\right)^{-1},
$$

where $\operatorname{vol}\left(S_{i}\right)=\Delta_{r} \Delta(i)$ is the area of the cell $S_{x}$. From (10), (12) and (13), we get

$$
D_{g}=\int \operatorname{vol}\left(S_{i}\right) \frac{1}{4} \frac{L L_{i}(r)}{m_{i} r_{\max }} \frac{1}{12}\left(\left(\frac{r_{\max }}{L}\right)^{2}+\left(\frac{2 r}{L_{i}(r)}\right)^{2}\right) p(x) d x .
$$

Using expression $p(x)=\frac{1}{2} e^{-2 r}$, we get

$$
D_{g}=\int_{-r}^{r} d u \int_{0}^{r_{\max }} \frac{1}{48}\left(\left(\frac{r_{\max }}{L}\right)^{2}+\left(\frac{2 r}{L_{i}(r)}\right)^{2}\right) e^{-2 r} d r,
$$

from which it follows that

$$
D_{g}=\frac{1}{24} \int_{0}^{r_{\max }}\left(\left(\frac{r_{\max }}{L}\right)^{2}+\left(\frac{2 r}{L_{i}(r)}\right)^{2}\right) r e^{-2 r} d r .
$$

For optimization, we use equation

$$
J=D_{g}(i)+\lambda \int_{0}^{r_{\max }} L_{i}(r) d r,
$$

where? is the Lagrange's multiplier. We get from (14) and (15)

$$
\begin{aligned}
& J=\frac{1}{24} \int_{0}^{r_{\max }}\left(\frac{r_{\max }}{L}\right)^{2} r e^{-2 r} d r+\frac{1}{24} \int_{0}^{r_{\max }}\left(\frac{2 r}{L_{i}(r)}\right)^{2} r e^{-2 r} d r \\
& +\lambda \int_{0_{i}}^{r_{\max }} L_{i}(r) d r .
\end{aligned}
$$

Now, we will find the partial derivative $\frac{\partial J}{\partial L_{i}(r)}=0$ in order to derive the optimal value for $L_{i}, L_{i o p t}$. We find that

$$
L_{i}(r)=\frac{r e^{-\frac{2}{3} r}}{\sqrt[3]{3 \lambda}} .
$$

Since we know that

$$
\begin{aligned}
& \sum_{i=1}^{L} L_{i}=N, \quad \sum_{i=1}^{L} L_{i} \Delta_{r}=\Delta_{r} N, \\
& \int_{0}^{r_{\max }} L_{i}(r) d r=N \frac{r_{\max }}{L}, \quad I=\int_{0}^{r_{\max }} r e^{-\frac{2}{3} r} d r,
\end{aligned}
$$

substituting (18) in (17) we find

$$
L_{i}(r)=N \frac{r_{\max }}{L} \frac{r e^{-\frac{2}{3} r}}{I(i)} .
$$

Returning (19) to (14), we get an expression for granular distortion as

$$
D_{g}=\frac{1}{24} \frac{r_{\max }^{2}}{L^{2}} I_{0}+\frac{1}{24} \frac{4 L^{2} I^{2}}{N^{2} r_{\max }^{2}},
$$

where is

$$
I_{0}=\int_{0}^{r_{\max }} r e^{-2 r} d r
$$

and $I$ is given by (18).

Evaluating

$$
\frac{\partial D_{g}}{\partial L}=0
$$

we find an expression for optimal $L$ as

$$
L_{o p t}=r_{\max } \sqrt[4]{\frac{I_{0} N^{2}}{4 I^{3}}},
$$

and returning (23) in (20) we find optimal granular distortion

$$
D_{g}=\frac{1}{6} \frac{I}{N} \sqrt{I_{0} I}=D_{g_{o p t}} .
$$

The overall distortion is given by

$$
D^{\left(r_{\max }\right)}=D_{g}+D_{\text {over }} \text {. }
$$

Minimizing $D^{\left(r_{\max }\right)}$ over $r_{\max }$ we find $r_{\max }^{o p t}$ for a fixed number of points $N$.

\section{An Example}

For different values of the number of points $N$ and optimal value for $r_{\max }$ (for which MSE is minimal), we will show how moment of inertia is changing by domains, then we will determine granular distortion by our method.

We will determine the optimal number of cells in every domain, taking nearest integer value. It is necessary to update the found values for $L_{i}$ (increase or decrease) in order for the total sum to be equal to $N / 4$. For the total cell number $N=256$, we have:

$$
\begin{aligned}
& N / 4=64, \quad r_{\max }=3.69, \quad L_{o p t}=10, \\
& L_{\text {iopt }}=\{2,6,7,8,8,8,7,7,6,5\} .
\end{aligned}
$$

The dimensions of cells and the medium lines of domains can be determined from

$$
\begin{aligned}
& \Delta_{r}=\frac{r_{\max }}{L_{o p t}}, \quad \Delta_{u}(i)=\frac{2 m_{i}}{L_{\text {iopt }}}, \\
& m_{i}=\frac{r_{\max }}{L_{\text {opt }}}\left(i-\frac{1}{2}\right), N_{i}=4 L_{i} .
\end{aligned}
$$

The calculated values, for $\Delta_{r}=0.369$, are given in Table 1.

For comparing the obtained results to the previous ones, $r_{\max }$ from (8) is used, being obtained for 1-D approach. The corresponding $D_{g}^{\text {scal }}$ is compared to the obtained result using the following gain definition $G=10 \log \left(D_{g}^{\text {scal }} / D_{g}^{\text {opt }}\right)$.

The gain obtained by our method over the uniform scalar quantization for rate $R=4\left(N=2^{2 R}\right)$ is 
$G=2.68 \mathrm{~dB}$. Decrease of distortion depends on point density.

Table 1.

\begin{tabular}{|c|c|c|c|c|}
\hline$I$ & $m_{i}$ & $\Delta_{u}(i)$ & $M\left(m_{i}\right)$ & $\frac{M\left(m_{i}\right)}{1 / 12}$ \\
\hline 1 & 0.1845 & 0.1845 & 1.1042 & 1.25 \\
\hline 2 & 0.5535 & 0.1845 & 0.1042 & 1.25 \\
\hline 3 & 0.9225 & 0.2635 & 0.0881 & 1.0571 \\
\hline 4 & 1.2915 & 0.3228 & 0.1841 & 1.0089 \\
\hline 5 & 1.6605 & 0.4151 & 0.0839 & 1.0068 \\
\hline 6 & 2.0295 & 0.5073 & 0.0876 & 1.051 \\
\hline 7 & 2.3985 & 0.6852 & 0.0998 & 1.1976 \\
\hline 8 & 2.7675 & 0.7907 & 0.1087 & 1.3044 \\
\hline 9 & 3.1365 & 1.0455 & 0.1328 & 1.5931 \\
\hline 10 & 3.5055 & 1.4022 & 0.1693 & 2.0316 \\
\hline
\end{tabular}

We will find an expression for point density for cases of square and rectangular cells, after that we will compare them. First, we will define an expression for point density for case of square cells. From (13), we get

$$
\lambda_{s}=\frac{L^{2}}{N\left(r_{\max }^{(s)}\right)^{2}}
$$

where $r_{\max }^{(s)}, r_{\max }$ are given for square cells. After defining values for $r_{\max }$ and $N$ we will first evaluate number of domain $L=\sqrt{N}=2^{R}$. For rectangular cells, we find from (13), (26) and (27)

$$
\lambda_{r}=\frac{L_{\text {opt }} L_{\text {iopt }}}{2 N m_{i}\left(r_{\max }^{(r)}\right)}=\frac{L_{\text {opt }}^{2} L_{\text {iopt }}}{2 N\left(r_{\max }^{(r)}\right)^{2}\left(i-\frac{1}{2}\right)} .
$$

From (28), (29) and expression $r_{\max }^{(r)}=r_{\max }^{(s)}$ it follows that

$$
\frac{\lambda_{r}}{\lambda_{s}}(i)=\frac{L_{o p t}^{2} L_{i o p t}}{2 L^{2}\left(i-\frac{1}{2}\right)} .
$$

From (30), we evaluate $\frac{\lambda_{r}}{\lambda_{s}}(i)$ for different $i$, as shown in Table 2 .

Table 2.

\begin{tabular}{|c|c|}
\hline$i$ & $\frac{\lambda_{r}}{\lambda_{s}}(i)$ \\
\hline 1 & 3.125 \\
\hline 2 & 3.125 \\
\hline 3 & 2.1875 \\
\hline 4 & 1.786 \\
\hline 5 & 1.389 \\
\hline 6 & 1.136 \\
\hline 7 & 0.841 \\
\hline 8 & 0.729 \\
\hline 9 & 0.551 \\
\hline 10 & 0.411 \\
\hline
\end{tabular}

If we compare rectangular and square cells, the gain of rectangular cells is possible with higher point density in the region of higher probability density function, what follows from Table 2 .

\section{Conclusion}

The analysis of Bennett's integral is given for optimal uniform quantization for two-dimensional Laplace source. This paper gives simple and complete analysis for constructing an optimal uniform twodimensional quantizer for Laplace source with optimal point density. We calculated granular distortion and observed a gain due to the usage of the optimal point density. Distortion depends on the moment of inertia and point density, and we gave those values tabularly. Based on the total gain, we can conclude that the effect of point density is dominated over the effect of moment of inertia. The loss of moment of inertia is less than the gain of point density in the region of higher probability density function. The moment of inertia of rectangular cells is significantly higher in the region of lower probability density function, because its effect to distortion is small. The point density is higher in the region of higher probability density function and its effect is dominated.

\section{References}

[1] D. Hui, D.L. Neuhoff. Asymptotic Analysis of Optimal Fixed-Rate Uniform Scalar Quantization. IEEE Trans., 2001, IT-47(3), 957-977.

[2] R.M. Gray, D.L.Neuhoff. Quantization. IEEE Trans., 1998, IT-44(6), 2325-2384.

[3] Na.S., D.L. Neuhoff. Bennett's Integral for Vector Quantizers. IEEE Transaction on Information Theory. July 1995, IT-41(4), 886-900.

[4] N.S. Jayant, P. Noll. Digital Coding of Waveforms. Principles and Applications to Speech and Video. Prentice-Hall, New Jersey (1984).

[5] Z.H. Peric, D. Drajic, Z.Nikolic. Asymptotic analysis of optimal uniform two-dimensional quantization for Laplace source. Electronics and Electrical Engineering, No.2(52), 2004, 5-8.

[6] Z.H. Peric, M.C. Stefanovic. Asymptotic Analysis of Optimal Uniform Polar Quantization. International Journal of Electronics and Communications, Vol.56, 2002, 345-347.

[7] D.A. Stojanovic, D.R. Aleksic, Z.H. Peric, A.Z. Jovanovic. Analisis of Uniform Polar Quantization over Bennet's Integral. Electronics and Electrical Engineering-Kaunas, No.8(64), 2005.

[8] D.G. Jeong, J. Gibson. Uniform and Piecewise Uniform Lattice Vector Quantization for Memoryless Gaussian and Laplacian Sources. IEEE Trans., 1993, IT-39(3), 786-804.

[9] W.R.Bennett. Spectra of quantized signals. BSTJ, Vol.27, July 1948, 446-472.

Received March 2006. 